\title{
Torsades de Pointes in Intravenous Drug Abuse-Associated Infective Endocarditis
}

\author{
Sarah Eapen, MD, ${ }^{1,2}$ Michael S. Firstenberg, MD, FACC, FAIM,,$^{2,3,4}$ \\ ${ }^{1}$ Northeast Ohio Medical University, Rootstown, OH, USA; ${ }^{2}$ Department of Surgery, Summa Akron City Hospital, Akron, \\ $\mathrm{OH}$, USA; ${ }^{3}$ Department of Surgery, The Medical Center of Aurora and Rose Hospital, Aurora, CO, USA; ${ }^{4} \mathrm{College}$ of \\ Osteopathic Medicine, Rocky Vista University, Parker, CO, USA;
}

\section{ABSTRACT}

Intravenous drug abuse rates in the United States have increased exponentially in recent years. Ohio is one of 5 states with the highest age-adjusted drug overdose death rates, with drug overdose causing 39.1 of every 100,000 deaths. In patients who survive, the associated morbidity poses a significant public health burden. Infective endocarditis, defined as an infection of the endocardium of the heart, is a potentially lethal consequence of bacteremia related to intravenous drug abuse. Methicillin-sensitive Staphylococcus aureus (MSSA) is the most commonly implicated organism and may affect the tricuspid valve. Indications for surgery include failure of medical management, worsening embolization, heart failure, and arrhythmias-typically bradyarrhythmias from infectious extension into the conduction system. Vegetation size and type of infection, such as drug-resistance pattern, fungal infection, and presence of prosthetic material, are becoming known risk factors for complications.

Studies have demonstrated that early surgery tends to have a better prognosis than delayed intervention. Established guidelines for right-sided surgery are slowly evolving. Tricuspid valve surgery is becoming more common in these cases, but there is known risk for heart block owing to proximity of the conduction system to the tricuspid valve annulus. If patients develop complete heart block postoperatively, pacemaker placement may be indicated. There may be reluctance to implantation because of the risk of device infection with infective endocarditis. In addition, many may recover conduction as infection and edema resolve. Generally, bradyarrhythmias are well tolerated. However, we present 2 cases of torsades de pointes related to post-tricuspidvalve-replacement bradyarrhythmia. Torsades de pointes is a potentially lethal form of polymorphic ventricular arrhythmia associated with QT interval prolongation. Post-tricuspid-valvereplacement mortality may be secondary to induced lethal ventricular arrhythmias.

Received August 23, 2018 ; received in revised form October 9, 2018; accepted October 9, 2018

Correspondence: Michael S Firstenberg, MD, FACC, FAIM, Chief, Cardiovascular and Cardiothoracic Surgery, The Medical Center of Aurora, 1444 S. Potomac, Suite 390, Aurora, CO 80012; 1-303-226-4650; fax: 1-303-751-6069 (e-mail: msfirst@gmail.com).

\section{INTRODUCTION}

The lifetime prevalence of drug abuse in the United States is 7.7 percent [Compton 2007]. Risk factors for abuse include male gender, younger age, and lower socioeconomic status [Compton 2007]. Only 8.1 percent of patients diagnosed with a drug abuse disorder will receive treatment [Compton 2007]. The highest age-adjusted drug overdose death rates in 2016 were reported in West Virginia, Ohio, New Hampshire, the District of Columbia, and Pennsylvania [Hedegaard 2017]. For every 100,000 deaths in Ohio, 39.1 are related to drug overdose [Hedegaard 2017]. The issue of drug abuse has become more prevalent over time, with a 21 percent increase in the age-adjusted drug overdose death rate from 2015 to 2016 [Hedegaard 2017].

Infective endocarditis is a potentially lethal complication of intravenous drug abuse. Methicillin-sensitive Staphylococcus aureus (MSSA) is the most commonly implicated organism and typically affects the tricuspid valve [Ji 2012]. Poorer outcomes are associated with infective endocarditis that develops secondary to methicillin-resistant Staphylococcus

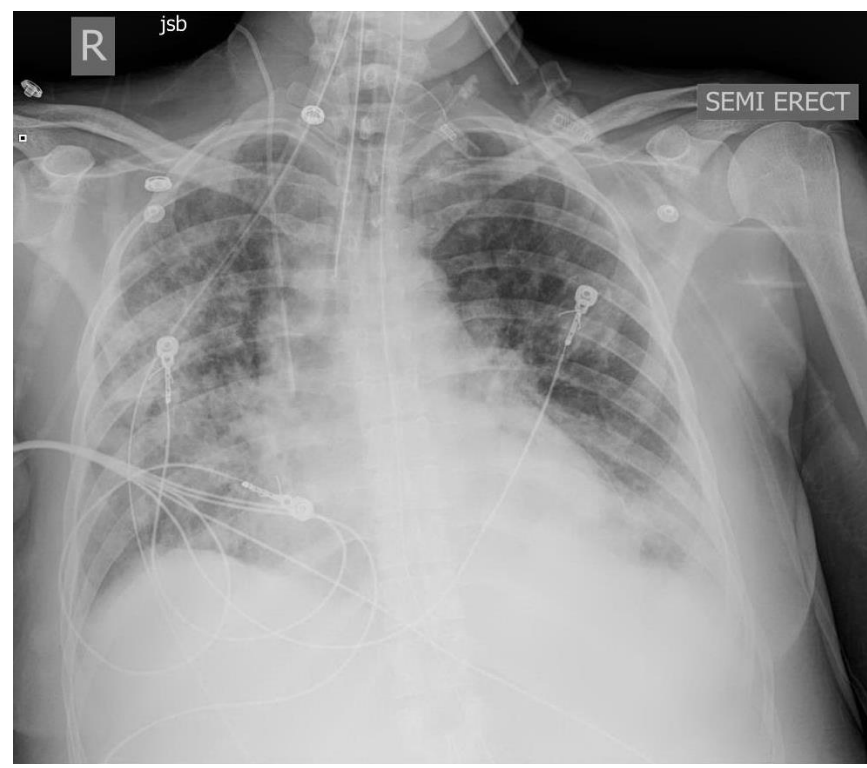

Figure 1. CXR with bilateral pulmonary infiltrates. 


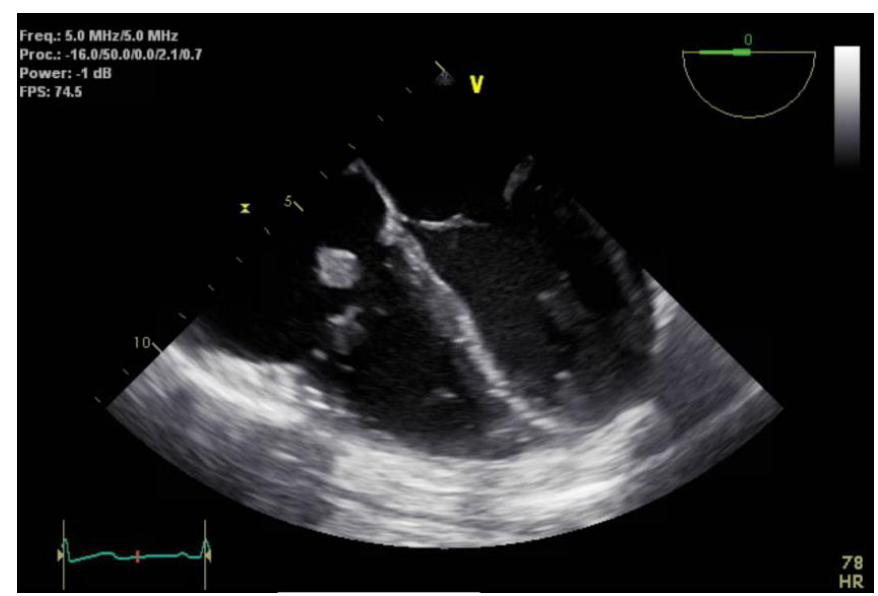

Figure 2. TEE with a large tricuspid vegetation consistent with tricuspid valve endocarditis.

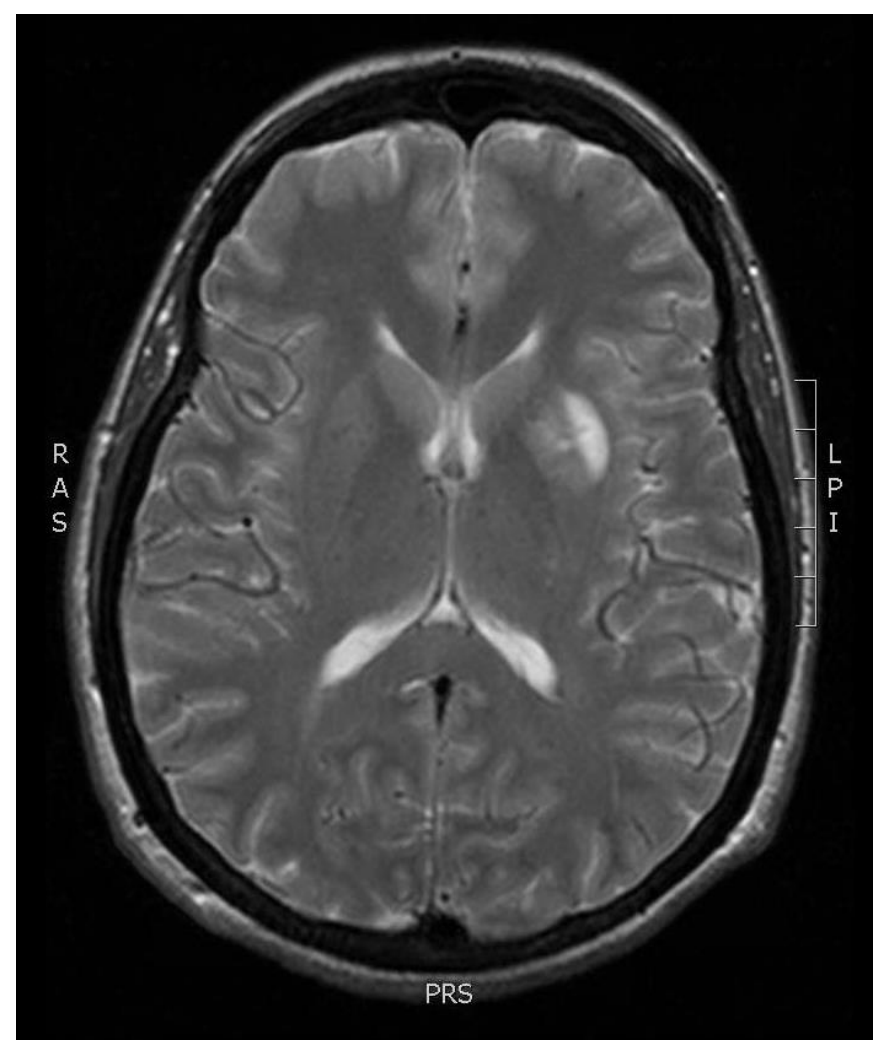

Figure 3. MRI of the brain with a left lentiform nucleus ischemic infarct.

aureus (MRSA), coagulase-negative staphylococcus, streptococcus, Pseudomonas aeruginosa, enterococcus, and HACEK organisms [Ji 2012]. Infective endocarditis is associated with higher rates of treatment failure and recurrence in intravenous drug abusers because of continued drug abuse [Ji 2012].

Early surgical intervention portends a better prognosis than delayed intervention. Indications for surgery include failure of medical management, worsening embolization,

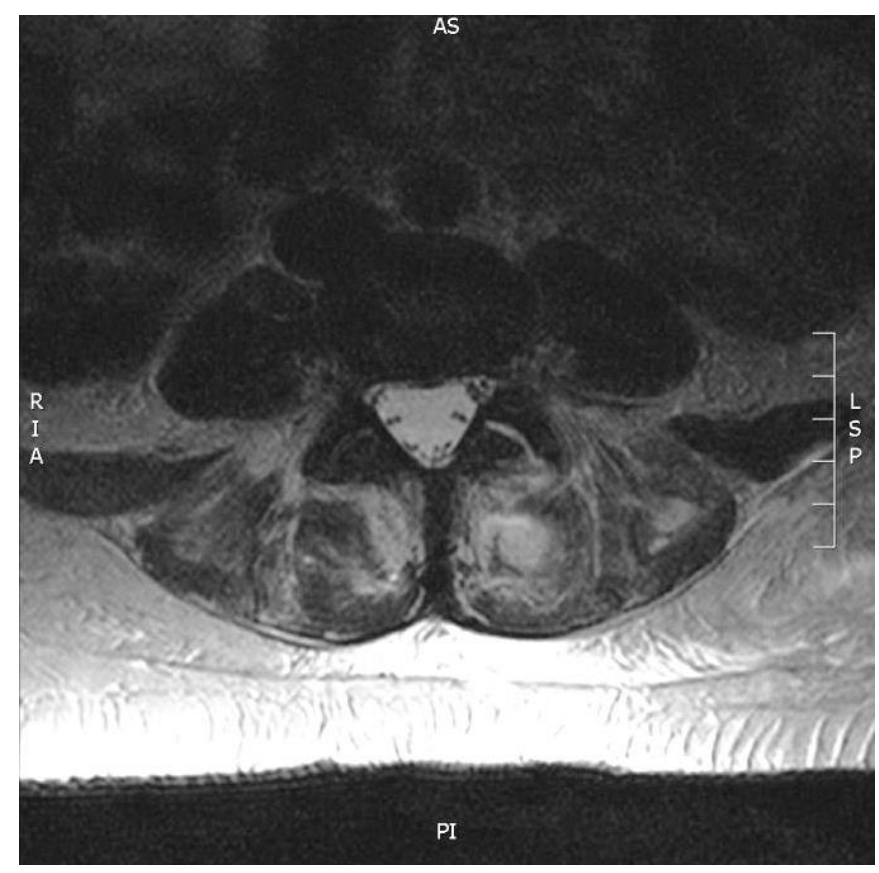

Figure 4. MRI of the spine with a left posterior paraspinal abscess.

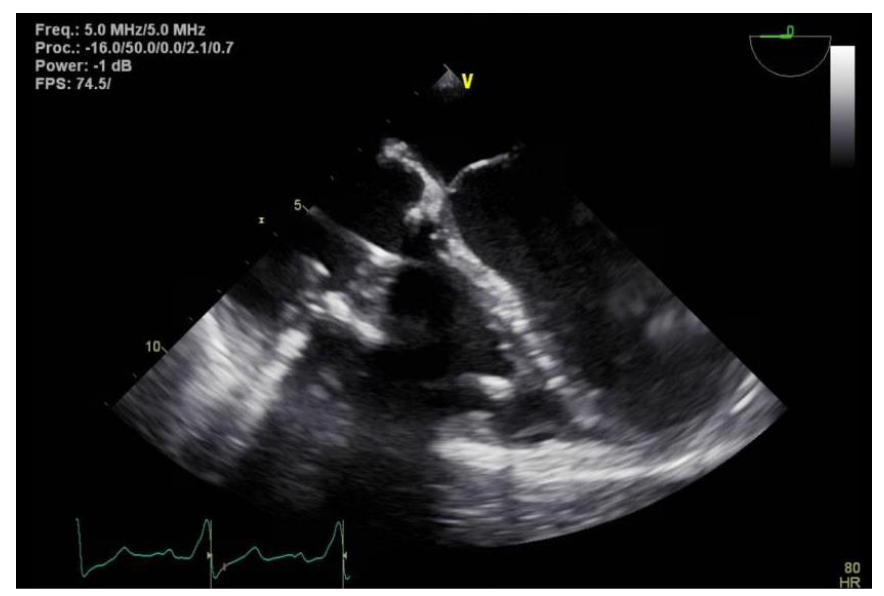

Figure 5. TEE with large vegetations of the tricuspid valve and rightventricular pacemaker lead.

heart failure, and arrhythmias. Bradyarrhythmias typically result from infectious extension into the conduction system. As infection and edema resolve, many may recover conduction. Tricuspid valve surgery itself poses risk for heart block because of proximity of the conduction system to the tricuspid valve annulus. Pacemaker placement may be indicated in cases of postoperative heart block. In cases of infective endocarditis, pacemaker implantation bears risk of device infection.

Although bradyarrhythmias are generally well tolerated, there is risk for progression to torsades de pointes. Torsades de pointes is a potentially lethal form of polymorphic ventricular arrhythmia associated with QT interval prolongation. We present 2 cases of torsades de pointes related to posttricuspid-valve-replacement bradyarrhythmia. 


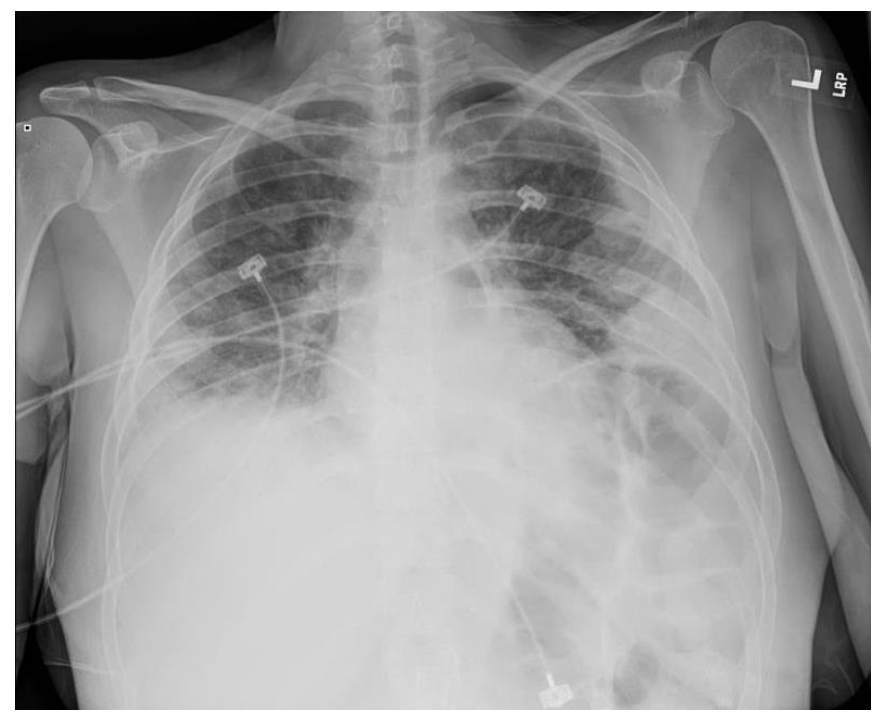

Figure 6. CXR with bilateral pulmonary infiltrates, left greater than right.

\section{CASE I}

A 33-year-old female with a history of methamphetamine abuse presented with fever, productive cough, and dyspnea. She was admitted with diagnoses of sepsis and MSSA bacteremia. Antibiotic therapy was initiated. Chest $\mathrm{x}$-ray (CXR) demonstrated bilateral pulmonary infiltrates (Figure 1). Transesophageal echocardiogram (TEE) revealed a large tricuspid vegetation consistent with tricuspid valve endocarditis (Figure 2). As a consequence of septic emboli, she developed a lentiform nucleus ischemic infarct (Figure 3), paraspinal abscess (Figure 4), and septic wrist and bilateral knee joints. She underwent tricuspid valve replacement with a 33-millimeter Medtronic tissue valve (Medtronic, Minneapolis, MN, USA), primary closure of an atrial septic defect, and bilateral acute pulmonary embolectomy. Selection of a tricuspid tissue valve was based on the patient's risk for noncompliance, eliminating the need for high-dose anticoagulation. Despite normal sinus rhythm in the early postoperative period, the patient went into complete heart block later in her postoperative course. Her heart block was attributed to inflammation surrounding the conduction system. With resolution of inflammation, resumed conduction was anticipated. For this reason, she was observed on antibiotic therapy.

During readmission for a groin incision seroma, she experienced 2 episodes of torsades de pointes. These episodes were exacerbated by periods of ventricular bigeminy. Initial transvenous pacemaker placement was followed by permanent dual-chamber-pacemaker placement. Following discharge, the patient required readmission for sepsis secondary to Haemophilus parainfluenzae bacteremia. TEE demonstrated large vegetations of her tricuspid valve and chronic right-ventricular pacemaker lead (Figure 5). She required cardiac decortication, implantation of a dual-chamber biventricular pacemaker, and tricuspid valve replacement with a 29-millimeter Medtronic tissue valve. She remained on antibiotic therapy and was discharged to a long-term acute care

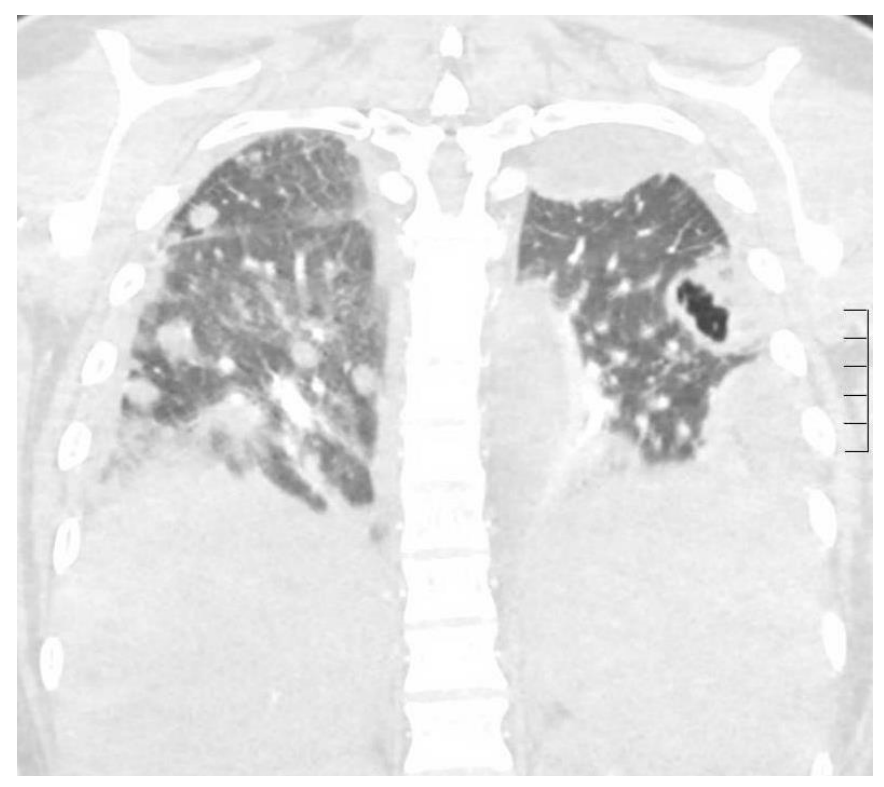

Figure 7. CTA of the chest with bilateral focal pulmonary densities and left-sided loculated pleural abscesses.

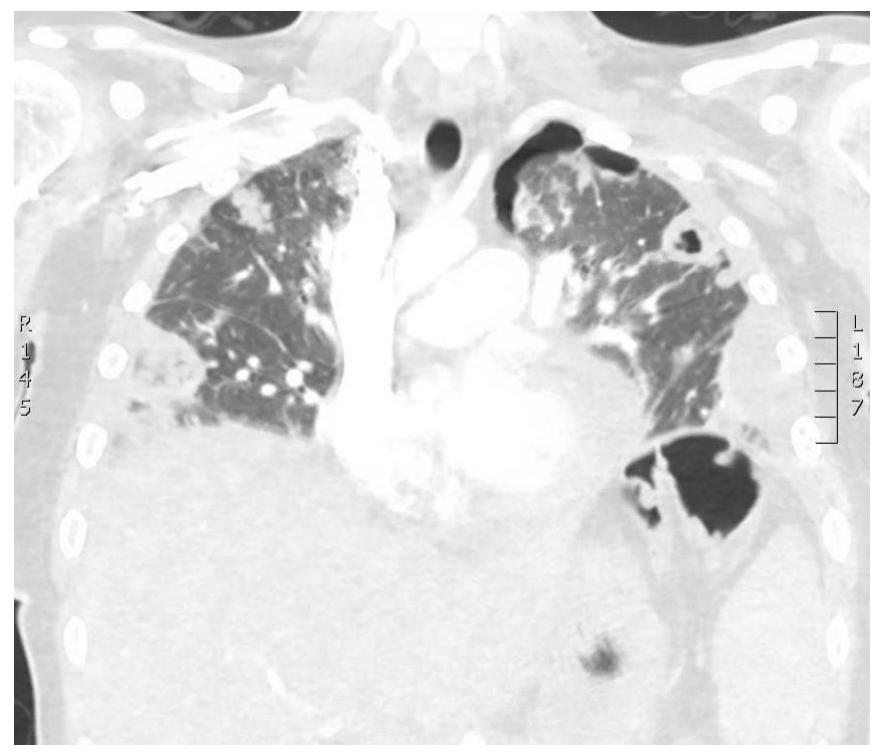

Figure 8. CTA of the chest with bilateral focal pulmonary densities and bilateral loculated pleural abscesses.

facility in stable condition. At 2-year follow-up, she remained free of infection without drug relapse. Recent device interrogation demonstrated pacemaker dependence, but no evidence of tachyarrhythmia.

\section{CASE 2}

A 25-year-old female with a history of hepatitis C and methamphetamine, heroin, and fentanyl abuse presented with generalized pain, malaise, and dyspnea. CXR demonstrated bilateral pulmonary infiltrates (Figure 6). Subsequent 


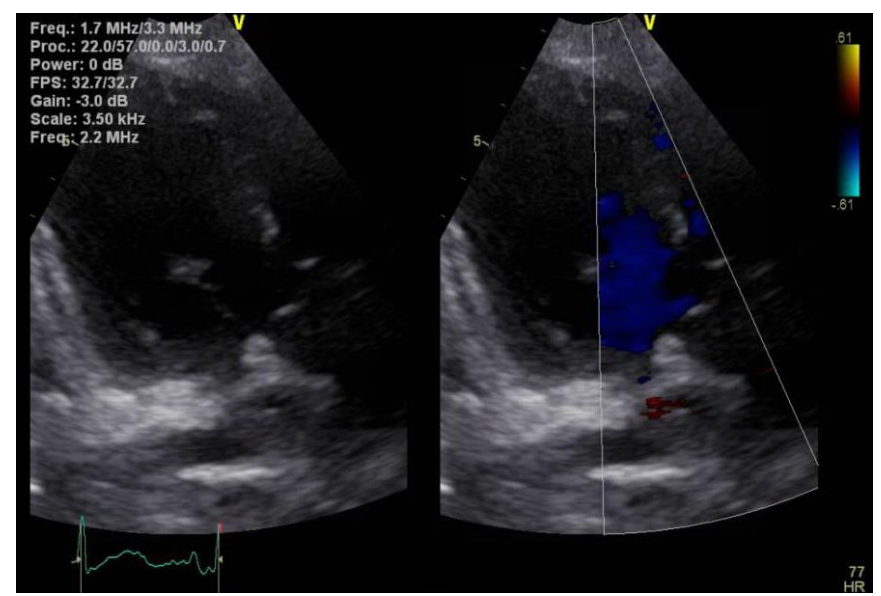

Figure 9. TEE with severe tricuspid regurgitation and large, mobile tricuspid vegetations.

computed tomography (CT) of the chest demonstrated bilateral focal pulmonary densities and loculated pleural abscesses (Figures 7-8). She was admitted with diagnoses of sepsis, MRSA bacteremia, and septic pulmonary emboli. Antibiotic therapy was initiated. TEE revealed severe tricuspid regurgitation and large, mobile tricuspid vegetations (Figure 9). She initially underwent a left thoracotomy with drainage of pleural abscess and left lower lobe wedge resection. Because of clinical progression, she subsequently underwent tricuspid valve replacement with a 29 -millimeter St. Jude tissue valve (Abbott Laboratories, Abbott Park, IL, USA) and right pulmonary decortication. Tricuspid tissue valve selection again eliminated the need for high-dose anticoagulation in a patient at risk for noncompliance. In the early postoperative period, she remained epicardial-lead-pacemaker dependent. The decision was made to defer permanent pacemaker placement unless symptomatic bradycardia developed.

Following discharge, she required readmission for symptomatic loculated pleural effusions (Figure 10). She underwent a right thoracotomy with complete decortication and wedge resections of right upper- and lower-lobe abscesses. Intraoperatively, she developed torsades de pointes and required defibrillation. She had several episodes of nonsustained torsades de pointes in the early postoperative period as well. Her polymorphic ventricular tachycardia was attributed to bradyarrhythmia. She subsequently underwent permanent pacemaker placement and was discharged to a long-term acute care facility in stable condition.

\section{DISCUSSION}

In cases of extension of infection into the cardiac septum, the conduction system may be affected, leading to arrhythmias [Mylonakis 2001]. Torsades de pointes is a form of polymorphic ventricular arrhythmia associated with QT interval prolongation [Tounsi 2012]. QT prolongation of 0.45 in males and 0.47 in females is diagnostic of long QT syndrome [Yuan 2009]. Acquired QT prolongation may be attributed to electrolyte abnormalities, such as hypokalemia, hypomagnesemia,

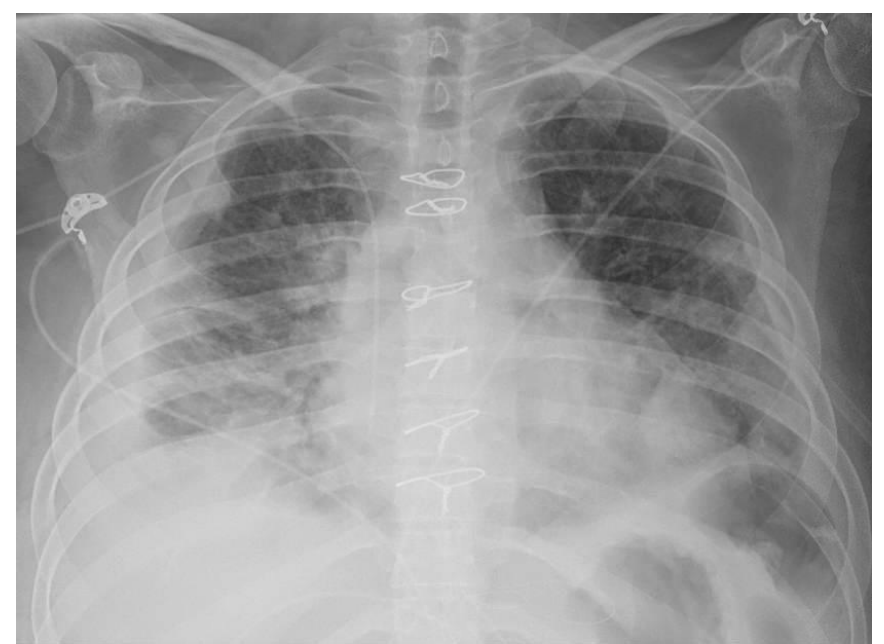

Figure 10. CXR with bilateral pleural effusions and patchy infiltrates

and hypocalcemia [Yuan 2009; Tounsi 2012]. Certain medications, such as calcium channel blockers, psychiatric medications, antihistamines, antibiotics, immunosuppressants, and diuretics may cause QT prolongation as well [Yap 2003; Yuan 2009]. Other causes of acquired QT prolongation include cocaine abuse, myocardial ischemia, subarachnoid hemorrhage, stroke, autonomic neuropathy, HIV infection, and chronic renal disease requiring dialysis [Khan 2002; Familoni 2006; Yuan 2009].

The majority of cases are pause-dependent, characterized by bradyarrhythmia or sinus pause causing QT interval prolongation and arrhythmia [Tounsi 2012]. In the cases described herein, the development of torsades de pointes was preceded by bradyarrhythmias, characteristic of the pausedependent subtype of QT prolongation. Magnesium sulfate is the treatment of choice for torsades de pointes [Hasegawa 1991]. Lidocaine may also be used [Hasegawa 1991]. Serum electrolyte abnormalities should be corrected, with serum potassium maintained at a high normal level [Hasegawa 1991; Yuan 2009]. If these treatments are ineffective, defibrillation is required [Tounsi 2012]. In addition, patients may require temporary transvenous pacing, which is specifically effective in pause-dependent QT prolongation [Koo 2004]. Febrile infection has been identified as a predisposing factor for QT prolongation and torsades de pointes [Tounsi 2012]. With completion of antibiotic therapy for infective endocarditis, resolution of arrhythmia has been described [Tounsi 2012].

Torsades de pointes is an uncommon presentation of infective endocarditis, with very few cases reported in the literature. Tounsi et al described a 49-year-old male with prosthetic mitral valve MRSA endocarditis who developed bradyarrhythmia, QT prolongation, and torsades de pointes [Tounsi 2012]. In this case, the patient's corrected QT interval was markedly prolonged on admission, leading to multiple episodes of torsades de pointes requiring cardiopulmonary resuscitation and defibrillation [Tounsi 2012]. His bradyarrhythmia, QT prolongation, and associated torsades de pointes resolved with completion of antibiotic therapy [Tounsi 2012]. 
Sayar et al reported a case of Brucella endocarditis affecting a prosthetic mitral valve in a 58-year-old female who developed QT prolongation and torsades de pointes [Sayar 2006]. Her QT interval was prolonged on admission, and she had multiple episodes of torsades de pointes requiring defibrillation thereafter [Sayar 2006]. She required reoperation and ultimately experienced arrhythmia resolution following completion of antibiotic therapy [Sayar 2006].

Yuan et al described a case of aortic and mitral valve Streptococcus viridans endocarditis complicating hypertrophic obstructive cardiomyopathy in a 52-year-old female who later developed first degree atrioventricular block and QT prolongation [Yuan 2009]. She required surgical intervention and experienced no further arrhythmias following completion of antibiotic therapy, beta-blockade, and potassium supplementation [Yuan 2009].

Finally, Irie et al reported a case of a 70-year-old male with infective endocarditis who developed QT prolongation and torsades de pointes secondary to hypoalbuminemia and propofol [Irie 2010]. The patient developed large, bilateral pleural effusions and required mechanical ventilation [Irie 2010]. After receiving a bolus of propofol for induction of sedation, he developed QT prolongation and torsades de pointes [Irie 2010]. His laboratory results were significant for hypoalbuminemia, attributed to malnutrition and increased catabolism related to his underlying infection [Irie 2010]. His arrhythmia resolved with beta-blockade and discontinuation of propofol. The patient's arrhythmia was attributed to the increased free propofol related to hypoalbuminemia and the effects of propofol on myocardial repolarization [Irie 2010].

The incidence of lead- and pocket-related complications associated with conventional transvenous pacemakers has given rise to the development of permanent leadless cardiac pacemakers. These devices are self-contained right-ventricular single-chamber pacemakers implanted by using a percutaneous femoral approach [Tjong 2017]. Leadless pacemakers comprise $15 \%$ to $30 \%$ of total pacemaker placement in Western countries [Mond 2001]. Indications for placement include chronic atrial fibrillation and atrioventricular block [Tjong 2017]. Short-term complication rates for leadless pacemakers are higher than complication rates for conventional pacemakers at $4.8 \%$ and $4.1 \%$, respectively [Tjong 2017]. Leadless pacemaker complications are often related to implantation and may be associated with the learning curve for the procedure [Tjong 2017]. These include femoral access site complications, cardiac perforation, and pacemaker dislodgement [Tjong 2017]. In comparison, short-term complications of conventional transvenous pacemaker placement include pneumothorax, pocket hematoma, cardiac tamponade, and lead dislodgement [Tjong 2017]. In addition, transvenous leads have been associated with venous obstruction, tricuspid regurgitation, and endocarditis [Tjong 2017]. Mortality rates for transvenous lead-related endocarditis range from $12 \%$ to $31 \%$ [Tjong 2017]. The comparison of leadless and conventional pacemakers is limited by the lack of long-term outcome data. Randomized controlled trials are needed to definitively determine the role of permanent leadless cardiac pacemaker therapy in clinical practice.

\section{CONCLUSION}

Intravenous drug abuse remains a significant public health issue in the United States. The development of infective endocarditis and its complications are associated with substantial morbidity and mortality. After the clinical diagnosis is made, antibiotic therapy is recommended for a duration of 4 to 6 weeks. Early surgery portends a better prognosis than delayed intervention. Torsades de pointes is a potentially lethal complication of infective endocarditis that may result from involvement of the cardiac conduction system. Tricuspid valve surgery also bears risk of heart block because of conduction system proximity to the tricuspid valve annulus. The initial approach to management addresses the underlying etiology of arrhythmia. If medical management is unsuccessful, defibrillation and transvenous pacing may be necessary. In patients with infective endocarditis presenting with QT prolongation, the potential risk for progression to torsades de pointes should be recognized early. Efforts should be made to identify and address potential inciting factors to prevent torsades de pointes as well. Although both patients in this report ultimately required permanent pacemaker placement, observation with temporary transvenous pacing and antibiotic therapy is a reasonable initial approach to management. Given the significant mortality rates of transvenous lead-related endocarditis, leadless pacemaker placement may provide a safe and effective alternative to conventional transvenous pacemaker placement. Randomized controlled trials are needed to define the role of leadless cardiac pacemaker therapy in the future.

\section{REFERENCES}

Compton WM, Thomas YF, Stinson FS, Grant BF. 2007. Prevalence, correlates, disability, and comorbidity of DSM-IV drug abuse and dependence in the United States. Arch Gen Psychiatry 64:566-576.

Familoni OB, Alebiosu CO, Ayodele OE. 2006. Effects and outcome of haemodialysis on QT intervals and QT dispersion in patients with chronic kidney disease. Cardiovasc J S Afr 17:19-23.

Hasegawa J, Takami T, Kaneda T, et al. 1991. Treatment of torsades de pointes with intravenous magnesium in idiopathic long QT syndrome. Jpn Circ J 55:1057-60.

Hedegaard H, Warner M, Minino AM. 2017. Drug overdose deaths in the United States, 1999-2016. Hyattsville (MD): National Center for Health Statistics. NCHS Data Brief no. 294.

Irie T, Kaneko Y, Nakajima T, Saito A, Kurabayashi M. 2010. QT interval prolongation and torsade de pointes induced by propofol and hypoalbuminemia. Int Heart J 51:365-6.

Ji Y, Kujtan L, Kershner D. 2012. Acute endocarditis in intravenous drug users: a case report and literature review. J Community Hosp Intern Med Perspect 2(1). doi: 10.3402/jchimp.v2i1.11513.

Khan IA. 2002. Long QT syndrome: diagnosis and management. Am Heart J 143:7-14.

Koo BN, Kang OC, Kim KB, Park WK, Kil HK. 2004. Anesthetic management of a patient with congenital long QT syndrome: a case report. Korean J Anesthesiol 46:743-6. Most of the paper is in Korean. 
Mond HG, Proclemer A. 2001. The 11th world survey of cardiac pacing and implantable cardioverter-defibrillators: calendar year 2009-a World Society of Arrhythmia's project. Pacing Clin Electrophysiol 34:1013-27.

Mylonakis E, Calderwood SB. Infective endocarditis in adults. 2001. N Engl J Med 345:1318-30.

Sayar N, Terzi S, Yilmaz HY, et al. 2006. A case of prosthetic mitral valve Brucella endocarditis complicated with torsades de pointes. Heart Vessels 21:331-3.

Tounsi A, Abid L, Akrout M, Hentati M, Kammoun S. 2012. QT prolongation complicated with torsades de pointes in prosthetic mitral valve endocarditis: a case report. Case Rep Med 2012:574923.

Tjong FV, Reddy VY. 2017. Permanent leadless cardiac pacemaker therapy: a comprehensive review. Circulation 135:1458-70.

Yap YG, Camm AJ. 2003. Drug induced QT prolongation and torsades de pointes. Heart 89:1363-72.

Yuan SM, Demosthenous E, Coman V. 2009. Long QT syndrome in extensive infective endocarditis complicating hypertrophic obstructive cardiomyopathy. Kardiol Pol;67:53-7. 